\title{
4. Análise dos itens de um trabalho científico ${ }^{1}$
}

\author{
Analysis of the topics of a scientific paper
}

Fabio Xerfan Nahas ${ }^{2}$, Lydia Masako Ferreira ${ }^{3}$

1. Programa de Pós-Graduação em Cirurgia Plástica da Universidade Federal de São Paulo - Escola Paulista de Medicina (UNIFESP-EPM). Disciplina de Cirurgia Plástica da UNIFESP-EPM.

2. Professor Adjunto Visitante da Disciplina de Cirurgia Plástica da UNIFESP-EPM. Professor Orientador do Programa de Pós-Graduação em Cirurgia Plástica da UNIFESP-EPM.

3. Professora Titular da Disciplina de Cirurgia Plástica da UNIFESP-EPM. Coordenadora do Programa de Pós-Graduação em Cirurgia Plástica da UNIFESP-EPM.

\section{RESUMO}

Os itens do trabalho científico são descritos e colocados de forma objetiva os respectivos conteúdos. O Título, a maior arma para seduzir o leitor, deve ser curto e informativo. A Introdução visa situar o leitor, mostrando, de modo objetivo e conciso, a relevância do estudo. O objetivo deve ser colocado ao final da Introdução como a pergunta base do trabalho. Em Métodos, foi reforçada a caracterização dos tipos mais utilizados de amostras em trabalho científico na área médica e discutida a descrição dos procedimentos. As diferentes maneiras de se expor os resultados e as preferências de acordo com o uso de tabela, tipos de gráficos são analisados. A lógica da discussão, respeitando a seqüência dos itens do trabalho e a comparação dos resultados com a literatura são descritos. O Resumo privilegiando a forma estruturada e a referências com sua posição no texto também são analisados.

Descritores: Artigo de Revista, Redação, Editoração, Métodos

\begin{abstract}
The items of a scientific paper are described and its contents are objectively analyzed. The title, the most powerful weapon to attract the reader should be short and bring up the information regarding the ideas within the article. The Introduction situates the reader, showing concisely the relevancy of the study. The purpose of the study - the main question that should be answered along the article - should be written at the end of the Introduction. In Methods, the most used types of samples in the medical field are reported and analyzed. The different ways to display the Results and the special forms to express the data such as the use of tables and graphics are analyzed. The logic of the Discussion, respecting the sequence of the items of a scientific paper and the results obtained by the author are compared with the literature data. The Abstract written by items (structured) and the References and its situation on the text are also analyzed.
\end{abstract}

Key words: Journal Article, Writing, Publishing, Methods

\section{Título}

É a maior arma para convencer o leitor de que vale a pena ler o artigo. Por esta razão deve ser curto e ao mesmo tempo completo, de fácil compreensão e traduzir a proposição do trabalho científico. O ideal é que, se possível, tenha apenas uma linha. Se houver dificuldade de se criar um título, pode-se coloca-lo na forma de pergunta, baseada no objetivo do trabalho. Este tipo de título traduz, em geral, a idéia do estudo e chama a atenção do leitor ${ }^{1}$.

\section{Introdução}

Visa situar o leitor acerca do que se passa na literatura mundial sobre o assunto. Deve ser concisa e objetiva, principalmente no caso de artigos para revistas. Neste item deve-se demonstrar a relevância do estudo, com citações e estatísticas, de forma bem resumida. No último parágrafo deve ser colocado o objetivo do trabalho. O objetivo é a pergunta à qual o estudo se propõe a responder e, ao final, deverá fazê-lo, seja positiva ou negativamente. A pergunta é geralmente única; entretanto, ao longo do trabalho, podem ser obtidas outras respostas, até mesmo inesperadas. A introdução e o resumo dão a primeira impressão ao revisor do periódico. Desta forma, merecem especial atenção do autor, pois aumentam a chance de aceitação do artigo.

\section{Métodos}

Neste item serão descritos a amostra e os procedimentos realizados durante o experimento ${ }^{2}$. Os detalhes que limitam e descrevem a amostra devem ser colocados neste momento. Quando são estudados animais, deve-se descrever o tipo (raça), sexo, faixa etária, etc... Em caso de cadáveres, sexo, idade, raça, local de obtenção e condição “post-mortem”, devem ser relatados. Quando material sintético é o foco do estudo, as normas técnicas, descrição da substância com o qual é feito e a referência da 
empresa que o produz, são dados fundamentais que devem ser descritos. Para pacientes, os dados básicos a serem relatados são sexo, idade, período em que o estudo foi realizado, tipo de seleção (ao acaso, consecutivos, etc...), a procedência (hospital, clínica, etc...).A distribuição dos pacientes nos grupos de estudo deve ser randomizada, o que nem sempre é fácil em estudos cirúrgicos ${ }^{3}$. Deve-se ainda caracterizar a amostra com índice de massa corpórea (IMC) em estudos de contorno corporal e medidas antropométricas.

\section{Descrição da amostra}

Algumas considerações e particularidades dos diferentes tipos de amostragem serão descritas a seguir, segundo o tipo de amostra:

$$
1 \text { - “In vitro” }
$$

O trabalho será um estudo "in vitro", quando os procedimentos não são realizados em seres vivos. São estudos realizados em laboratório e que envolvem materiais sintéticos ou ainda estudos com células, toxinas ou bactérias. Este é um tipo de estudo não muito freqüente em cirurgia plástica, entretanto, próteses de silicone, de poliuretano, materiais biocompatíveis podem ser testados em laboratório. Em geral são pesquisas realizadas em conjunto com profissionais especializados em bioengenharia. Estudos “in vitro" podem também ser realizados com produtos biológicos como a toxina botulínica, cultura de tecidos, fatores de crescimento e terapia gênica (relacionados à biologia molecular).

\section{2-Animais}

No caso de estudo com animais, deve-se estar atento ao modelo experimental a ser selecionado. Deve-se considerar que alguns animais, dependendo da idade, podem crescer. Nestes casos, estudos que necessitem de medidas lineares ou de áreas (retalhos, áreas doadoras de enxertia, expansores, etc...) terão os parâmetros finais alterados. Existem animais cujo tecido celular subcutâneo é mínimo e, geralmente localizado na região inguinal (roedores, por exemplo). Desta forma, para se estudar lipoaspiração, alguns tipos de porcos são bons modelos experimentais. Em relação ao espaço subcutâneo, é importante ressaltar que quase todos os mamíferos têm uma estrutura denominada “paniculum carnosum”. Esta estrutura é uma camada de músculo lamelar localizada sob a pele, cuja função é contração rápida após o ferimento. A finalidade funcional do “paniculum carnosum” é diminuir hemorragia e reduzir a área cruenta, encurtando o tempo da cicatrização por $2^{\mathrm{a}}$ intenção. Nos seres humanos, ao longo da evolução, esta camada involuiu e temos remanescentes equivalentes como o SMAS, platisma e provavelmente a fáscia de Scarpa. Animais que apresentam "paniculum carnosum” não são modelos adequados para estudo de cicatrização ou queimaduras, pois os parâmetros são perdidos após o procedimento. Outros fatores devem ser levados em conta quando animais são utilizados. Existem comissões que defendem os direitos dos animais, cuja ação tende a crescer no Brasil. Nos Estados Unidos estas comissões são formadas por veterinários, médicos e membros de associações de defesa dos animais. Naquele país é permitido que cada animal seja submetido a apenas uma intervenção ou experimento. Essas comissões julgam se o tipo de experimento não leva ao sofrimento do animal em questão e até discutem sobre o método do experimento. Quanto mais desenvolvido na escala biológica, maior a resistência destes grupos a estudo experimental em animais. Protocolos com macacos, cães e gatos têm maior dificuldade de aprovação. O porte do animal tem relação direta com o custo do experimento. Animais de pequeno porte (camundongos, ratos, cobaias) são mais baratos, exigem menos espaço e anestesia pode ser intraperitoneal, tornando a presença de técnico desnecessária. Já animais grandes (porcos, cães e macacos) necessitam de acomodações maiores e anestesia geral; têm, portanto custo mais elevado. Deve-se também levar em conta a agressividade de cada animal. Cobaias e camundongos são mais dóceis e fáceis de trabalhar. Alguns tipos de ratos são mais agressivos. Deve-se separar os animais em janelas distintas no pós-operatório para evitar ataque às incisões ou feridas pós-operatórias. Existe também a chance de que alguns animais podem evoluir com óbito, portanto deve-se superestimar o número de animais no projeto de pesquisa. A escolha do animal a ser estudado pode ser baseada em estudos semelhantes encontrados na literatura.

\section{3 - Cadáveres}

A grande vantagem de se utilizar cadáveres em estudos é o fato de não haver necessidade de seguimento, entretanto o principal desafio é encontrar o que ainda não foi estudado em anatomia. Para os cirurgiões plásticos há limites de dissecção que, em geral, devem ser respeitados em cadáveres frescos (face, mãos e pernas). O tempo "postmortem” determina o grau de deterioração e rigidez do cadáver e deve ser considerado dependendo do tipo do estudo. O cadáver deve ser selecionado por faixa etária e sexo, de acordo com o grupo que se quer estudar. Por exemplo, em um estudo sobre as alterações da gestação na parede abdominal, cadáveres de homens de 70 a 80 anos não podem ser selecionados.

\section{4 - Pacientes}

Estudos em pacientes têm sido cada vez mais valorizados por periódicos ${ }^{4}$. A seleção de pacientes deve ser realizada baseada em qual grupo a pesquisa aplica-se. Por exemplo, ao selecionar 100 pacientes de 30 anos para estudar proporções faciais e aplicá-las pacientes que desejam submeter-se à face-lift cuja idade média seja 55 anos. Este trabalho terá sido prejudicado pelo erro no planejamento do método. Outro exemplo semelhante é estudar pacientes que não tenham tido filho aplicando estes conhecimentos para casos de abdominoplastia. Ou seja, a amostra tem que ser significativa do universo na qual será aplicada a tese. A seleção ao acaso, de pacientes consecutivos, geralmente diminui a possibilidade de erro, quando bem aplicada. Por exemplo, estudar as 20 próximas pacientes que serão submetidas a abdominoplastia, em 
geral, em média estas devem ter tido dois filhos e estar na faixa etária de 30 a 45 anos, o que será representativo do universo no qual as conclusões poderão ser aplicadas. Desta foram, idade, sexo e a forma de seleção são fatores importantes. A vantagem de se estudar pacientes é que estes são, em geral, o modelo experimental ideal ${ }^{4}$. Entretanto deve-se respeitar os limites éticos, o que pode limitar a gama de métodos a serem utilizados, limitando assim as conclusões. O principal obstáculo neste tipo de estudo é o seguimento, pois as pessoas podem mudar-se ou perde-se o contato por alteração de endereço, telefone ou ainda podem simplesmente não querer voltar.

\section{Métodos (descrição dos procedimentos)}

Os métodos devem ter, como características fundamentais, viabilidade, reprodutibilidade e simplicidade. Para tal, deve-se sempre que possível fazer um plano piloto. O plano piloto consiste em testar, com alguns indivíduos, animais, cadáveres, ou materiais aloplásticos o que planejamos realizar nos métodos. Estes testes poderão ou não ser inclusos no trabalho. O plano piloto tem como finalidade verificar possíveis falhas do método e melhoralo, ou até para verificar a viabilidade deste. Todo valor que possa ter variações quando medido deve ser verificado por mais de um observador. Se forem dois observadores, o método deve prever desempate (talvez a inclusão de um terceiro observador) a não ser que o método estatístico dispense o desempate. A avaliação pode ser realizada por médicos e, em cirurgia estética, por leigos (até pelo próprio paciente). A descrição do método deve ser detalhada para que este possa ser reproduzível. Tratando-se de tese, esta descrição deve ser colocada nos mínimos detalhes, sem se preocupar com o número de palavras. Já, em publicações para periódicos, este item deve ser o máximo descritivo e conciso ao mesmo tempo.

\section{Análise estatística}

No final do método deve-se descrever a análise estatística empregada. Existem basicamente dois grandes grupos de testes estatísticos: 1) paramétricos (mais conhecidos); 2) não paramétricos (não tão utilizados). Se o método for não habitual, algumas revistas solicitam que seja descrita sua metodologia. Deve-se, sempre que possível, utilizar métodos conhecidos. Um profissional da estatística deve ser envolvido no estudo para evitar erros. Embora existam programas que executam cálculos estatísticos, se houver erro na elaboração ou interpretação dos testes, pode repercutir nas conclusões do estudo. O pesquisador deve estar atento a resultados estatísticos que não tenham valor clínico. Estes resultados devem ser excluídos do trabalho ou devem ser citados como dados sem validade clínica.

\section{Resultados}

Os resultados devem ser relatados com clareza e podem ser expostos de três modos: A) no texto, para valores únicos ou poucos dados; B) em tabelas que facilitam a apresentação de alguns tipos de dados. No caso de números com casa decimal ou centesimal, os dados são melhores visualizados que quando apresentados na forma de gráficos; C) em gráficos que podem dar a noção de evolução, comparação e frações de um total. Os valores são facilmente notados quando há utilização correta dos gráficos, sensibilizando o leitor. Os resultados não devem ser repetidos no trabalho em suas diversas formas de apresentação. Se os valores forem colocados na tabela, os mesmos não devem ser apresentados em gráficos. Toda tabela e gráfico deve ter título auto-explicativo. As tabelas têm grande apelo visual. Podem conter palavras (quadros), números ou dados estatísticos. As tabelas são utilizadas para resumir os resultados e não para colocar todos os dados obtidos. Existe uma regra prática na qual aproximadamente 1 tabela deve ter relação com aproximadamente 1000 palavras. Existem vários tipos de gráficos, entretanto os mais utilizados são:

1) Gráfico Linear (abscissa/ordenada). Podem dar a idéia de evolução (crescimento, redução). É também indicado em estudos mais complexos para representar diversas variáveis;

2) Gráfico de Barras, pode ser utilizado para dar idéia de evolução. Gráfico com múltiplas barras (duplas, triplas) pode ser utilizado para dar idéia de comparação entre grupos, associado à evolução no tempo, por exemplo. Neste caso é melhor que o gráfico linear, pois facilita a percepção do leitor. É geralmente utilizado com as barras na posição vertical. Pode também ser representado com as barras colocadas horizontalmente quando se pretende demonstrar relações de causa e efeito.

3) Gráfico de Setores (pizza), é ideal para representar frações. Indicado para apresentar a distribuição por sexo, idade, localização de lesões, etc...

Toda a tabela e gráfico deve ter título conciso e claro. O tamanho da amostra também deve ser colocado (valor de “n”). O teste estatístico utilizado, o desvio padrão e o valor de p (ou equivalente) deve ser sempre referido, qualquer que seja a forma de exposição dos resultados (em tabelas, gráficos ou no texto). No item Resultados, os valores devem ser apenas relatados e nunca comentados ou justificados. A interpretação dos resultados deve ser colocada no item Discussão.

\section{Discussão}

A discussão é essência do artigo científico. Expressa as opiniões dos autores em relação ao tema em estudo ${ }^{6}$ e permite comparações dos resultados obtidos com os dados disponíveis na literatura. Existe uma ordem bem definida que torna sua compreensão mais fácil, entretanto, erroneamente, nem sempre é seguida em trabalhos científicos. Deve-se iniciar pela discussão da introdução. Nesta fase é discutido, em profundidade, o cenário atual do problema estudado, citando a importância da aplicação prática do estudo. A seguir deve-se discutir o porquê da escolha do material ou casuística. No caso de estudo com animais, por quais motivos o animal em questão foi selecionado. No caso de cadáveres, o que levou o pesquisador a utiliza-los e quais suas vantagens em relação a seres vivos (estudos musculares podem variar com 
anestésicos, sendo de difícil avaliação em seres vivos). Cada item do método utilizado na avaliação em questão deve ser comentado. Após esta análise, os resultados obtidos devem ser discutidos e analisados. Os valores obtidos devem ser confrontados com dados da literatura e estas comparações devem ser discutidas. A discussão continua com a expressão livre dos autores em relação aos resultados e seus potenciais desdobramentos e aplicações. É neste momento que o autor pode discorrer com maior liberdade sobre o tema, fazendo suas hipóteses e considerações. São colocados os futuros caminhos a serem estudados sobre o assunto ${ }^{7}$.

\section{Conclusão}

Atualmente alguns periódicos como o Plastic and Reconstructive Surgery e o Annals of Plastic Surgery incluem a conclusão como último parágrafo da discussão, sem coloca-la em item separado. Obrigatoriamente os autores devem concluir com a resposta à questão inicial do trabalho colocada no objetivo. Todo trabalho deve ter pelo menos esta conclusão. Entretanto, outras conclusões podem ter sido obtidas durante o estudo ${ }^{8}$.

\section{Resumo / Abstract}

O resumo deve ser elaborado somente após ter sido escrito todo o trabalho. Coloca-se o máximo de detalhes com a maior objetividade possível. Deve ser respeitada a seqüência dos itens do trabalho. Resume-se, desta forma, a introdução, métodos, resultados, discussão e conclusão. Todos os itens devem estar presentes. É de extrema importância, pois tem a função de seduzir o leitor a ler o artigo em sua totalidade. É importante lembrar que o resumo é a única parte de seu estudo que será lida pela maioria dos leitores ${ }^{9}$. Em casos de publicações em periódicos de língua não inglesa, em geral é solicitado que o resumo seja também escrito em inglês. Este texto deverá observar a maior fidelidade à língua inglesa possível. Sua compreensão será fundamental para citações futuras em revistas internacionais.

\section{Referências}

Apenas as referências bibliográficas citadas no texto devem ser listadas neste item. Todo periódico tem sua sistemática de colocar as referências e agrupá-las. Alguns solicitam que as referências sejam colocadas por ordem alfabética e outros de acordo com a entrada no texto. As referências bibliográficas, com raras exceções, devem estar presentes apenas na introdução e discussão. Quando é utilizado algum método citado ou descrito em outro artigo, pode haver a necessidade de colocar-se alguma referência na secção de Métodos. Existem programas que coletam a bibliografia da internet, formatando-a de acordo com a instrução para autores da revista escolhida e coloca a referência no texto.

\section{Referências}

1. Fishbein M. Medical writing. 3rd. ed. York: McGrawHill; 1957.

2. Montenegro MR. Metodologia do trabalho científico. Material e métodos. In:Gonçalves EL, editor. Pesquisa médica. 1st. ed. São Paulo: EPU- Conselho Nacional de Desenvolvimento Científico e Tecnológico; 1983.p.111-109.

3. Ferreira L M. Surgical randomized controlled trials: reflection of the difficulties. Acta Cir Bras. 2004 19(l):2-3.

4. Goldenberg S, Goldenberg A, Ferreira L M, Montero EFS. Pesquisa clínica na Acta Cirúrgica Brasileira. Acta Cir Bras. 200520(4):270-271.

5. Nahas FX, Ferreira LM, Sabino Neto M, Garcia EB. Elaboração de trabalho científico. Rev Bras Cir Plast. 2004 19(2):11-28.

6. Rigatto M. A discussão dos resultados: a hipótese, a síntese, a tese. In:Gonçalves EL, editor. Pesquisa médica. 1st. ed. São Paulo: EPU- Conselho Nacional de Desenvolvimento Científico e Tecnológico; 1983.p.124-108.

7. Rey L. Como redigir trabalhos científicos. 1st. ed. São Paulo: Editora Edgard Blücher (Editora da Universidade de São Paulo); 1972.

8. Liebano RE, Dias SL, Ferreira LM. Number of objectives and conclusions in dissertations and thesis. Acta Cir Bras. 2005 20(4):272-272.

9. Lilleyman JS. How to write a scientific paper-a rough guide to getting published. Arch Dis Child. 1995; 72(3):268-70.

\section{Correspondência:}

Fabio Xerfan Nahas

Disciplina de Cirurgia Plástica / Departamento de Cirurgia

Universidade Federal de São Paulo (UNIFESP - EPM)

Rua Napoleão de Barros, 715 - $4^{\circ}$ andar

04024-900 São Paulo-SP Brasil

Tel: (5511) 5576-4118

nahas.dcir@epm.br

\section{Como citar este artigo:}

Nahas FX, Ferreira LM. Análise dos itens de um trabalho científico. Acta Cir Bras [periódico na Internet] 2005;20 Suppl. 2:13-6. Disponível em URL: http://www.scielo.br/acb 